\title{
Bilinear Operators on Normed Linear Spaces
}

\author{
Kazuhisa Nakasho \\ Yamaguchi University \\ Yamaguchi, Japan
}

\begin{abstract}
Summary. The main aim of this article is proving properties of bilinear operators on normed linear spaces formalized by means of Mizar [1]. In the first two chapters, algebraic structures $[3$ of bilinear operators on linear spaces are discussed. Especially, the space of bounded bilinear operators on normed linear spaces is developed here. In the third chapter, it is remarked that the algebraic structure of bounded bilinear operators to a certain Banach space also constitutes a Banach space.

In the last chapter, the correspondence between the space of bilinear operators and the space of composition of linear opearators is shown. We referred to [4, 11, 2, 7] and 8 in this formalization.
\end{abstract}

$\begin{array}{lllll}\text { MSC: 46-00 47A07 47A30 68T99 03B35 } & \end{array}$

Keywords: Lipschitz continuity; bounded linear operator; bilinear operator; algebraic structure; Banach space

MML identifier: LOPBAN_9, version: 8.1.09 5.54.1341

\section{Real Vector Space of Bilinear Operators}

Let $X, Y, Z$ be real linear spaces. The functor $\operatorname{BilinOpers}(X, Y, Z)$ yielding a subset of RealVectSpace((the carrier of $X \times Y), Z)$ is defined by

(Def. 1) for every set $x, x \in$ it iff $x$ is a bilinear operator from $X \times Y$ into $Z$.

Let us observe that $\operatorname{BilinOpers}(X, Y, Z)$ is non empty and functional and $\operatorname{BilinOpers}(X, Y, Z)$ is linearly closed.

The functor VectorSpaceOfBilinOpers $\mathbb{R}_{\mathbb{R}}(X, Y, Z)$ yielding a strict RLS structure is defined by the term 
(Def. 2) $\langle\operatorname{BilinOpers}(X, Y, Z)$, Zero(BilinOpers $(X, Y, Z)$, RealVectSpace((the carrier of $X \times Y), Z)$ ), $\operatorname{Add}(\operatorname{BilinOpers}(X, Y, Z)$, RealVectSpace((the carrier of $X \times Y), Z)$ ), Mult(BilinOpers $(X, Y, Z)$, RealVectSpace((the carrier of $X \times Y), Z))\rangle$.

Let us note that VectorSpaceOfBilinOpers $\mathbb{R}_{\mathbb{R}}(X, Y, Z)$ is non empty and VectorSpaceOfBilinOpers $\mathbb{R}_{\mathbb{R}}(X, Y, Z)$ is Abelian, add-associative, right zeroed, right complementable, vector distributive, scalar distributive, scalar associative, and scalar unital and VectorSpaceOfBilinOpers $\mathbb{R}_{\mathbb{R}}(X, Y, Z)$ is constituted functions.

Now we state the proposition:

(1) Let us consider real linear spaces $X, Y, Z$. Then VectorSpaceOfBilin$\operatorname{Opers}_{\mathbb{R}}(X, Y, Z)$ is a subspace of RealVectSpace((the carrier of $\left.\left.X \times Y\right), Z\right)$.

Let $X, Y, Z$ be real linear spaces, $f$ be an element of VectorSpaceOfBilin$\operatorname{Opers}_{\mathbb{R}}(X, Y, Z), v$ be a vector of $X$, and $w$ be a vector of $Y$. Let us note that the functor $f(v, w)$ yields a vector of $Z$. Now we state the propositions:

(2) Let us consider real linear spaces $X, Y, Z$, and vectors $f, g, h$ of Vector$\operatorname{SpaceOfBilinOpers}_{\mathbb{R}}(X, Y, Z)$. Then $h=f+g$ if and only if for every vector $x$ of $X$ and for every vector $y$ of $Y, h(x, y)=f(x, y)+g(x, y)$.

(3) Let us consider real linear spaces $X, Y, Z$, vectors $f, h$ of VectorSpaceOf$\operatorname{BilinOpers}_{\mathbb{R}}(X, Y, Z)$, and a real number $a$. Then $h=a \cdot f$ if and only if for every vector $x$ of $X$ and for every vector $y$ of $Y, h(x, y)=a \cdot f(x, y)$.

Let us consider real linear spaces $X, Y, Z$. Now we state the propositions:

(4) $0_{\text {VectorSpaceOfBilinOpers }}(X, Y, Z)=($ the carrier of $X \times Y) \longmapsto 0_{Z}$.

(5) (The carrier of $X \times Y) \longmapsto 0_{Z}$ is a bilinear operator from $X \times Y$ into $Z$.

\section{Real Normed Linear Space of Bounded Bilinear Operators}

Let $X, Y, Z$ be real normed spaces and $I_{1}$ be a bilinear operator from $X \times$ $Y$ into $Z$. We say that $I_{1}$ is Lipschitzian if and only if

(Def. 3) there exists a real number $K$ such that $0 \leqslant K$ and for every vector $x$ of $X$ and for every vector $y$ of $Y,\left\|I_{1}(x, y)\right\| \leqslant K \cdot\|x\| \cdot\|y\|$.

Now we state the propositions:

(6) Let us consider real normed spaces $X, Y, Z$, and a bilinear operator $f$ from $X \times Y$ into $Z$. Suppose for every vector $x$ of $X$ for every vector $y$ of $Y, f(x, y)=0_{Z}$. Then $f$ is Lipschitzian.

(7) Let us consider real normed spaces $X, Y, Z$. Then (the carrier of $X \times$ $Y) \longmapsto 0_{Z}$ is a bilinear operator from $X \times Y$ into $Z$. 
Let $X, Y, Z$ be real normed spaces. Let us observe that there exists a bilinear operator from $X \times Y$ into $Z$ which is Lipschitzian.

Now we state the proposition:

(8) Let us consider real normed spaces $X, Y, Z$, and an object $z$. Then $z \in \operatorname{BilinOpers}(X, Y, Z)$ if and only if $z$ is a bilinear operator from $X \times$ $Y$ into $Z$.

Let $X, Y, Z$ be real normed spaces. The functor BoundedBilinOpers $(X, Y, Z)$ yielding a subset of VectorSpaceOfBilinOpers $\mathbb{R}(X, Y, Z)$ is defined by

(Def. 4) for every set $x, x \in i t$ iff $x$ is a Lipschitzian bilinear operator from $X \times$ $Y$ into $Z$.

Note that BoundedBilinOpers $(X, Y, Z)$ is non empty and BoundedBilinOpers $(X, Y, Z)$ is linearly closed.

The functor VectorSpaceOfBoundedBilinOpers $\mathbb{R}_{\mathbb{R}}(X, Y, Z)$ yielding a strict RLS structure is defined by the term

(Def. 5) 〈BoundedBilinOpers $(X, Y, Z)$, Zero(BoundedBilinOpers $(X, Y, Z)$, VectorSpaceOfBilinOpers $\left._{\mathbb{R}}(X, Y, Z)\right), \operatorname{Add}(\operatorname{BoundedBilinOpers}(X, Y, Z)$,

VectorSpaceOfBilinOpers $\left._{\mathbb{R}}(X, Y, Z)\right)$, Mult(BoundedBilinOpers $(X, Y, Z)$, VectorSpaceOfBilinOpers $(X, Y, Z))\rangle$.

Now we state the proposition:

(9) Let us consider real normed spaces $X, Y, Z$. Then VectorSpaceOfBounded$\operatorname{BilinOpers}_{\mathbb{R}}(X, Y, Z)$ is a subspace of VectorSpaceOfBilinOpers $\mathbb{R}_{\mathbb{R}}(X, Y, Z)$.

Let $X, Y, Z$ be real normed spaces. Note that VectorSpaceOfBoundedBilin$\operatorname{Opers}_{\mathbb{R}}(X, Y, Z)$ is non empty and VectorSpaceOfBoundedBilinOpers $\mathbb{R}_{\mathbb{R}}(X, Y, Z)$ is Abelian, add-associative, right zeroed, right complementable, vector distributive, scalar distributive, scalar associative, and scalar unital and VectorSpaceOfBoundedBilinOpers $\mathbb{R}_{\mathbb{R}}(X, Y, Z)$ is constituted functions.

Let $f$ be an element of VectorSpaceOfBoundedBilinOpers $\mathbb{R}(X, Y, Z), v$ be a vector of $X$, and $w$ be a vector of $Y$. One can verify that the functor $f(v, w)$ yields a vector of $Z$. Now we state the propositions:

(10) Let us consider real normed spaces $X, Y, Z$, and vectors $f, g, h$ of VectorSpaceOfBoundedBilinOpers $_{\mathbb{R}}(X, Y, Z)$. Then $h=f+g$ if and only if for every vector $x$ of $X$ and for every vector $y$ of $Y, h(x, y)=f(x, y)+$ $g(x, y)$. The theorem is a consequence of $(2)$.

(11) Let us consider real normed spaces $X, Y, Z$, vectors $f$, $h$ of VectorSpaceOfBoundedBilinOpers $_{\mathbb{R}}(X, Y, Z)$, and a real number $a$. Then $h=a \cdot f$ if and only if for every vector $x$ of $X$ and for every vector $y$ of $Y, h(x, y)=$ $a \cdot f(x, y)$. The theorem is a consequence of $(3)$.

(12) Let us consider real normed spaces $X, Y, Z$. 
Then $0_{\text {VectorSpaceOfBoundedBilinOpers }}(X, Y, Z)=($ the carrier of $X \times Y) \longmapsto 0_{Z}$.

The theorem is a consequence of (4).

Let $X, Y, Z$ be real normed spaces and $f$ be an object. Assume $f \in$ BoundedBilinOpers $(X, Y, Z)$. The functor modetrans $(f, X, Y, Z)$ yielding a Lipschitzian bilinear operator from $X \times Y$ into $Z$ is defined by the term

(Def. 6) $f$.

Let $u$ be a bilinear operator from $X \times Y$ into $Z$. The functor $\operatorname{PreNorms}(u)$ yielding a non empty subset of $\mathbb{R}$ is defined by the term

(Def. 7) $\{\|u(t, s)\|$, where $t$ is a vector of $X, s$ is a vector of $Y:\|t\| \leqslant 1$ and $\|s\| \leqslant 1\}$.

Let $g$ be a Lipschitzian bilinear operator from $X \times Y$ into $Z$. Observe that PreNorms $(g)$ is upper bounded.

Now we state the proposition:

(13) Let us consider real normed spaces $X, Y, Z$, and a bilinear operator $g$ from $X \times Y$ into $Z$. Then $g$ is Lipschitzian if and only if $\operatorname{PreNorms}(g)$ is upper bounded.

Let $X, Y, Z$ be real normed spaces. The functor BoundedBilinOpersNorm $(X$, $Y, Z)$ yielding a function from BoundedBilinOpers $(X, Y, Z)$ into $\mathbb{R}$ is defined by

(Def. 8) for every object $x$ such that $x \in \operatorname{BoundedBilinOpers}(X, Y, Z)$ holds it $(x)=\sup \operatorname{PreNorms}(\operatorname{modetrans}(x, X, Y, Z))$.

Let $f$ be a Lipschitzian bilinear operator from $X \times Y$ into $Z$. Let us note that modetrans $(f, X, Y, Z)$ reduces to $f$.

Now we state the proposition:

(14) Let us consider real normed spaces $X, Y, Z$, and a Lipschitzian bilinear operator $f$ from $X \times Y$ into $Z$. Then (BoundedBilinOpersNorm $(X, Y, Z)$ ) $(f)=\sup \operatorname{PreNorms}(f)$.

Let $X, Y, Z$ be real normed spaces. The functor NormSpaceOfBoundedBilin$\operatorname{Opers}_{\mathbb{R}}(X, Y, Z)$ yielding a non empty normed structure is defined by the term

(Def. 9) 〈BoundedBilinOpers $(X, Y, Z)$, Zero(BoundedBilinOpers $(X, Y, Z)$, VectorSpaceOfBilinOpers $\left._{\mathbb{R}}(X, Y, Z)\right)$, Add(BoundedBilinOpers $(X, Y, Z)$, VectorSpaceOfBilinOpers $\left._{\mathbb{R}}(X, Y, Z)\right)$, Mult(BoundedBilinOpers $(X, Y, Z)$, VectorSpaceOfBilinOpers $\left._{\mathbb{R}}(X, Y, Z)\right)$, BoundedBilinOpersNorm $\left.(X, Y, Z)\right\rangle$.

Now we state the propositions:

(15) Let us consider real normed spaces $X, Y, Z$. Then (the carrier of $X \times$ $Y) \longmapsto 0_{Z}=0_{\text {NormSpaceOfBoundedBilinOpers }}(X, Y, Z)$. The theorem is a consequence of (12). 
(16) Let us consider real normed spaces $X, Y, Z$, a point $f$ of NormSpaceOfBoundedBilinOpers $_{\mathbb{R}}(X, Y, Z)$, and a Lipschitzian bilinear operator $g$ from $X \times Y$ into $Z$. Suppose $g=f$. Let us consider a vector $t$ of $X$, and a vector $s$ of $Y$. Then $\|g(t, s)\| \leqslant\|f\| \cdot\|t\| \cdot\|s\|$. The theorem is a consequence of (14).

Let us consider real normed spaces $X, Y, Z$ and a point $f$ of NormSpaceOfBoundedBilinOpers $_{\mathbb{R}}(X, Y, Z)$. Now we state the propositions:

(17) $0 \leqslant\|f\|$. The theorem is a consequence of (14).

(18) If $f=0_{\text {NormSpaceOfBoundedBilinOpers }_{\mathbb{R}}(X, Y, Z)}$, then $0=\|f\|$. The theorem is a consequence of (15) and (14).

Let $X, Y, Z$ be real normed spaces. One can verify that every element of NormSpaceOfBoundedBilinOpers $_{\mathbb{R}}(X, Y, Z)$ is function-like and relation-like.

Let $f$ be an element of NormSpaceOfBoundedBilinOpers $\mathbb{R}_{\mathbb{R}}(X, Y, Z), v$ be a vector of $X$, and $w$ be a vector of $Y$. Observe that the functor $f(v, w)$ yields a vector of $Z$. Now we state the propositions:

(19) Let us consider real normed spaces $X, Y, Z$, and points $f, g, h$ of NormSpaceOfBoundedBilinOpers $_{\mathbb{R}}(X, Y, Z)$. Then $h=f+g$ if and only if for every vector $x$ of $X$ and for every vector $y$ of $Y, h(x, y)=$ $f(x, y)+g(x, y)$. The theorem is a consequence of $(10)$.

(20) Let us consider real normed spaces $X, Y, Z$, points $f$, $h$ of NormSpaceOfBoundedBilinOpers $_{\mathbb{R}}(X, Y, Z)$, and a real number $a$. Then $h=a \cdot f$ if and only if for every vector $x$ of $X$ and for every vector $y$ of $Y, h(x, y)=$ $a \cdot f(x, y)$. The theorem is a consequence of (11).

(21) Let us consider real normed spaces $X, Y, Z$, points $f, g$ of NormSpaceOfBoundedBilinOpers $_{\mathbb{R}}(X, Y, Z)$, and a real number $a$. Then

(i) $\|f\|=0$ iff $f=0_{\mathrm{NormSpaceOfBoundedBilinOpers}_{\mathbb{R}}(X, Y, Z)}$, and

(ii) $\|a \cdot f\|=|a| \cdot\|f\|$, and

(iii) $\|f+g\| \leqslant\|f\|+\|g\|$.

Proof: $\|f+g\| \leqslant\|f\|+\|g\| \cdot\|a \cdot f\|=|a| \cdot\|f\|$.

Let $X, Y, Z$ be real normed spaces. Observe that NormSpaceOfBoundedBilin$\operatorname{Opers}_{\mathbb{R}}(X, Y, Z)$ is non empty and NormSpaceOfBoundedBilinOpers $\mathbb{R}(X, Y, Z)$ is reflexive, discernible, and real normed space-like.

Now we state the proposition:

(22) Let us consider real normed spaces $X, Y, Z$. Then NormSpaceOfBounded$\operatorname{BilinOpers}_{\mathbb{R}}(X, Y, Z)$ is a real normed space.

Let $X, Y, Z$ be real normed spaces. Let us note that NormSpaceOfBounded$\operatorname{BilinOpers}_{\mathbb{R}}(X, Y, Z)$ is vector distributive, scalar distributive, scalar associati- 
ve, scalar unital, Abelian, add-associative, right zeroed, and right complementable.

Now we state the proposition:

(23) Let us consider real normed spaces $X, Y, Z$, and points $f, g, h$ of NormSpaceOfBoundedBilinOpers $_{\mathbb{R}}(X, Y, Z)$. Then $h=f-g$ if and only if for every vector $x$ of $X$ and for every vector $y$ of $Y, h(x, y)=$ $f(x, y)-g(x, y)$. The theorem is a consequence of (19).

\section{Real Banach Space of Bounded Bilinear Operators}

Now we state the propositions:

(24) Let us consider real normed spaces $X, Y, Z$. Suppose $Z$ is complete. Let us consider a sequence $s_{1}$ of NormSpaceOfBoundedBilinOpers $\mathbb{R}_{\mathbb{R}}(X, Y, Z)$. If $s_{1}$ is Cauchy sequence by norm, then $s_{1}$ is convergent.

Proof: Define $\mathcal{P}$ [set, set] $\equiv$ there exists a sequence $x_{3}$ of $Z$ such that for every natural number $n, x_{3}(n)=v \operatorname{veq}(n)\left(\$_{1}\right)$ and $x_{3}$ is convergent and $\$_{2}=\lim x_{3}$. For every element $x_{4}$ of $X \times Y$, there exists an element $z$ of $Z$ such that $\mathcal{P}\left[x_{4}, z\right]$. Consider $f$ being a function from the carrier of $X \times$ $Y$ into the carrier of $Z$ such that for every element $z$ of $X \times Y, \mathcal{P}[z, f(z)]$. Reconsider $t_{1}=f$ as a function from $X \times Y$ into $Z$. For every points $x_{1}$, $x_{2}$ of $X$ and for every point $y$ of $Y, t_{1}\left(x_{1}+x_{2}, y\right)=t_{1}\left(x_{1}, y\right)+t_{1}\left(x_{2}, y\right)$. For every point $x$ of $X$ and for every point $y$ of $Y$ and for every real number $a, t_{1}(a \cdot x, y)=a \cdot t_{1}(x, y)$. For every point $x$ of $X$ and for every points $y_{1}$, $y_{2}$ of $Y, t_{1}\left(x, y_{1}+y_{2}\right)=t_{1}\left(x, y_{1}\right)+t_{1}\left(x, y_{2}\right)$.

For every point $x$ of $X$ and for every point $y$ of $Y$ and for every real number $a, t_{1}(x, a \cdot y)=a \cdot t_{1}(x, y) . t_{1}$ is Lipschitzian by [6, (18)], [9, (20)], (16). For every real number $e$ such that $e>0$ there exists a natural number $k$ such that for every natural number $n$ such that $n \geqslant k$ for every point $x$ of $X$ for every point $y$ of $Y$, $\| v \operatorname{seq}(n)(x, y)-$ $t_{1}(x, y)\|\leqslant e \cdot\| x\|\cdot\| y \|$ by [10, (8)], (23). Reconsider $t_{2}=t_{1}$ as a point of NormSpaceOfBoundedBilinOpers $\mathbb{R}(X, Y, Z)$. For every real number $e$ such that $e>0$ there exists a natural number $k$ such that for every natural number $n$ such that $n \geqslant k$ holds $\left\|v \operatorname{seq}(n)-t_{2}\right\| \leqslant e$. For every real number $e$ such that $e>0$ there exists a natural number $m$ such that for every natural number $n$ such that $n \geqslant m$ holds $\left\|v \operatorname{seq}(n)-t_{2}\right\|<e$.

(25) Let us consider real normed spaces $X, Y$, and a real Banach space $Z$. Then NormSpaceOfBoundedBilinOpers $\mathbb{R}_{\mathbb{R}}(X, Y, Z)$ is a real Banach space. The theorem is a consequence of (24). 
Let $X, Y$ be real normed spaces and $Z$ be a real Banach space. Let us note that NormSpaceOfBoundedBilinOpers $_{\mathbb{R}}(X, Y, Z)$ is complete.

\section{Isomorphisms Between the Space of Bilinear Operators and the Space of Composition of Linear Operators}

From now on $X, Y, Z$ denote real linear spaces.

Now we state the proposition:

(26) There exists a linear operator $I$ from VectorSpaceOfLinearOpers $\operatorname{Vip}_{\mathbb{R}}(X$, VectorSpaceOfLinearOpers $\left._{\mathbb{R}}(Y, Z)\right)$ into $\operatorname{VectorSpaceOfBilinOpers}_{\mathbb{R}}(X, Y$, $Z$ ) such that

(i) $I$ is bijective, and

(ii) for every point $u$ of VectorSpaceOfLinearOpers $\mathbb{R}_{\mathbb{R}}(X$, VectorSpaceOf$\left.\operatorname{LinearOpers}_{\mathbb{R}}(Y, Z)\right)$ and for every point $x$ of $X$ and for every point $y$ of $Y, I(u)(x, y)=u(x)(y)$.

Proof: Set $X_{1}=$ the carrier of $X$. Set $Y_{1}=$ the carrier of $Y$. Set $Z_{1}=$ the carrier of $Z$. Consider $I_{0}$ being a function from $\left(Z_{1}^{Y_{1}}\right)^{X_{1}}$ into $Z_{1} X_{1} \times Y_{1}$ such that $I_{0}$ is bijective and for every function $f$ from $X_{1}$ into $Z_{1}^{Y_{1}}$ and for every objects $d$, e such that $d \in X_{1}$ and $e \in Y_{1}$ holds $I_{0}(f)(d, e)=f(d)(e)$. Set $L_{1}=$ the carrier of VectorSpaceOfLinearOpers $\mathbb{R}_{\mathbb{R}}\left(X, \operatorname{VectorSpaceOf}_{\text {- }}\right.$ $\left.\operatorname{LinearOpers}_{\mathbb{R}}(Y, Z)\right)$. Set $B=$ the carrier of $\operatorname{VectorSpaceOfBilinOpers} \mathbb{R}_{\mathbb{R}}(X$, $Y, Z)$. Reconsider $I=I_{0}\left\lceil L_{1}\right.$ as a function from $L_{1}$ into $Z_{1} X_{1} \times Y_{1}$.

For every element $x$ of $L_{1}$, for every point $p$ of $X$ and for every point $q$ of $Y$, there exists a linear operator $G$ from $Y$ into $Z$ such that $G=x(p)$ and $I(x)(p, q)=G(q)$ and $I(x) \in B$. For every elements $x_{1}, x_{2}$ of $L_{1}, I\left(x_{1}+x_{2}\right)=I\left(x_{1}\right)+I\left(x_{2}\right)$. For every element $x$ of $L_{1}$ and for every real number $a, I(a \cdot x)=a \cdot I(x)$. For every point $u$ of VectorSpaceOfLinearOpers $\mathbb{R}_{\mathbb{R}}\left(X, \operatorname{VectorSpaceOfLinearOpers}_{\mathbb{R}}(Y, Z)\right)$ and for every point $x$ of $X$ and for every point $y$ of $Y, I(u)(x, y)=u(x)(y)$. For every object $y$ such that $y \in B$ there exists an object $x$ such that $x \in L_{1}$ and $y=I(x)$.

In the sequel $X, Y, Z$ denote real normed spaces.

(27) There exists a linear operator $I$ from the real norm space of bounded linear operators from $X$ into the real norm space of bounded linear operators from $Y$ into $Z$ into NormSpaceOfBoundedBilinOpers $\mathbb{R}(X, Y, Z)$ such that

(i) $I$ is bijective, and 
(ii) for every point $u$ of the real norm space of bounded linear operators from $X$ into the real norm space of bounded linear operators from $Y$ into $Z,\|u\|=\|I(u)\|$ and for every point $x$ of $X$ and for every point $y$ of $Y, I(u)(x, y)=u(x)(y)$.

Proof: Set $X_{1}=$ the carrier of $X$. Set $Y_{1}=$ the carrier of $Y$. Set $Z_{1}=$ the carrier of $Z$. Consider $I_{0}$ being a function from $\left(Z_{1}^{Y_{1}}\right)^{X_{1}}$ into $Z_{1} X_{1} \times Y_{1}$ such that $I_{0}$ is bijective and for every function $f$ from $X_{1}$ into $Z_{1}^{Y_{1}}$ and for every objects $d$, e such that $d \in X_{1}$ and $e \in Y_{1}$ holds $I_{0}(f)(d, e)=f(d)(e)$. Set $L_{1}=$ the carrier of the real norm space of bounded linear operators from $X$ into the real norm space of bounded linear operators from $Y$ into $Z$. Set $B=$ the carrier of NormSpaceOfBoundedBilinOpers $\operatorname{NR}_{\mathbb{R}}(X, Y, Z)$. Set $L_{2}=$ the carrier of the real norm space of bounded linear operators from $Y$ into $Z$. $L_{2}{ }^{X_{1}} \subseteq\left(Z_{1}^{Y_{1}}\right)^{X_{1}}$. Reconsider $I=I_{0}\left\lceil L_{1}\right.$ as a function from $L_{1}$ into $Z_{1} X_{1} \times Y_{1}$.

For every element $x$ of $L_{1}$, for every point $p$ of $X$ and for every point $q$ of $Y$, there exists a Lipschitzian linear operator $G$ from $Y$ into $Z$ such that $G=x(p)$ and $I(x)(p, q)=G(q)$ and $I(x)$ is a Lipschitzian bilinear operator from $X \times Y$ into $Z$ and $I(x) \in B$ and there exists a point $I_{2}$ of $\operatorname{NormSpaceOfBoundedBilinOpers} \mathbb{R}(X, Y, Z)$ such that $I_{2}=I(x)$ and $\|x\|=\left\|I_{2}\right\|$. For every elements $x_{1}, x_{2}$ of $L_{1}, I\left(x_{1}+x_{2}\right)=I\left(x_{1}\right)+I\left(x_{2}\right)$. For every element $x$ of $L_{1}$ and for every real number $a, I(a \cdot x)=a \cdot I(x)$. For every point $u$ of the real norm space of bounded linear operators from $X$ into the real norm space of bounded linear operators from $Y$ into $Z$, $\|u\|=\|I(u)\|$ and for every point $x$ of $X$ and for every point $y$ of $Y$, $I(u)(x, y)=u(x)(y)$. For every object $y$ such that $y \in B$ there exists an object $x$ such that $x \in L_{1}$ and $y=I(x)$ by [5. (12)].

Acknowledgement: I would like to express my gratitude to Professor Yasunari Shidama for his helpful advice.

\section{REFERENCES}

[1] Grzegorz Bancerek, Czesław Byliński, Adam Grabowski, Artur Korniłowicz, Roman Matuszewski, Adam Naumowicz, and Karol Pąk. The role of the Mizar Mathematical Library for interactive proof development in Mizar. Journal of Automated Reasoning, 61(1):9-32, 2018. do1:10.1007/s10817-017-9440-6

[2] Nelson Dunford and Jacob T. Schwartz. Linear operators I. Interscience Publ., 1958.

[3] Adam Grabowski, Artur Korniłowicz, and Christoph Schwarzweller. On algebraic hierarchies in mathematical repository of Mizar. In M. Ganzha, L. Maciaszek, and M. Paprzycki, editors, Proceedings of the 2016 Federated Conference on Computer Science and Information Systems (FedCSIS), volume 8 of Annals of Computer Science and Information Systems, pages 363-371, 2016. doi $10.15439 / 2016$ F520.

[4] Miyadera Isao. Functional Analysis. Riko-Gaku-Sya, 1972. 
[5] Kazuhisa Nakasho, Yuichi Futa, and Yasunari Shidama. Continuity of bounded linear operators on normed linear spaces. Formalized Mathematics, 26(3):231-237, 2018. doi: 10.2478 /forma-2018-0021

[6] Hiroyuki Okazaki, Noboru Endou, and Yasunari Shidama. Cartesian products of family of real linear spaces. Formalized Mathematics, 19(1):51-59, 2011. doi:10.2478/v10037011-0009-2.

[7] Laurent Schwartz. Théorie des ensembles et topologie, tome 1. Analyse. Hermann, 1997.

[8] Laurent Schwartz. Calcul différentiel, tome 2. Analyse. Hermann, 1997.

[9] Yasunari Shidama. Banach space of bounded linear operators Formalized Mathematics, 12(1):39-48, 2004.

[10] Yasumasa Suzuki, Noboru Endou, and Yasunari Shidama. Banach space of absolute summable real sequences Formalized Mathematics, 11(4):377-380, 2003.

[11] Kosaku Yoshida. Functional Analysis. Springer, 1980.

Accepted February 27, 2019 